\title{
Elecciones y paridad de género en Bolivia ${ }^{1}$
}

\section{Elections and gender parity in Bolivia}

María Inés Tula ${ }^{2}$

Resumen

El presente trabajo tiene como objetivo describir las reglas electorales y la aplicación de la paridad de género en Bolivia y su eficacia en el acceso a la representación. Para ello se analizan las elecciones del 18 de octubre de 2020 y sus resultados electorales y en la participación política de las mujeres en la Asamblea Legislativa Plurinacional.

Palabras clave: paridad vertical; paridad horizontal; participación política; acceso a la representación

\begin{abstract}
The present work aims to describe electoral rules and the application of gender parity in Bolivia and its effectiveness in access to representation. For this, the elections of october 18, 2020 and their electoral results and the political participation of women in the Asamblea Legislativa Plurinacional are analyzed.
\end{abstract}

Keywords: vertical parity; horizontal parity; political participation; access to representation

\section{Introducción}

La inclusión tardía de las mujeres a la ciudadanía a través del reconocimiento al derecho del sufragio activo no derivó en un aumento de las mujeres en las listas electorales, tampoco en cargos partidarios jerárquicos para la toma de decisiones. Como las reglas y procedimientos establecidos en los procesos de selección de candidatos impactan tanto en la estructura interna partidaria como en la oferta electoral, se buscó un mecanismo que nivelara las históricas diferencias de género (Krook, 2006; Choque Aldana, 2013; Johnson, 2014; Archenti y Tula, 2017).

\footnotetext{
1 Trabajo recibido el 31/03/2021. Aceptado el 01/06/2021. Este trabajo es una versión más acotada y ligeramente distinta de la presentada como Informe Final de la Misión de la Misión de Observación Electoral de la Organización de los Estados Americanos. La autora participó en carácter de especialista en Participación Política de las Mujeres durante las elecciones celebradas el 18 de octubre de 2020 en Bolivia.

${ }^{2}$ Universidad de Buenos Aires. Consejo Nacional de Investigaciones Científicas y Técnicas. Contacto: inestula@yahoo.com
} 
Así, las cuotas de género primero y la paridad después se sancionaron en los diferentes países de América Latina para superar los obstáculos que impedían a las mujeres ingresar a la política en iguales condiciones que los hombres. Si bien existen múltiples y variadas razones que condicionan esta posibilidad, tales como el contexto social, la educación, factores económicos, étnicos, políticos y culturales, el ejercicio de los derechos muchas veces se ve impedido por la acción combinada de otras complejas prácticas tales como los prejuicios y ciertos sistemas de creencias sociales que profundizan la desigualdad estructural existente (Saba, 2016).

En un sentido amplio la paridad es entendida como un nuevo contrato social entre mujeres y hombres. Está orientado a transformar las desigualdades persistentes en todos los ámbitos de la vida en sociedad, tanto en el ámbito público como en el privado (más bien, doméstico). En su acepción más restringida, la paridad electoral supone un equilibrio entre mujeres y hombres al interior de las organizaciones políticas y en la oferta partidaria. El modo en que esta última se articula es a través de la inclusión de un porcentaje equitativo (50\%) de varones y mujeres junto con una distribución alternada y secuencial (uno a uno) en toda su extensión. Ello permite: 1) producir entornos igualitarios en la estructuración de la oferta (inter-intra) partidaria en la competencia electoral, 2) visibilizar y naturalizar la participación de mujeres en cargos expectables de decisión de modo frecuente y no como casos aislados (Tula, 2017).

El presente trabajo presenta un análisis de las elecciones llevadas adelante en Bolivia durante los comicios de 2020. Interesa este país porque fue uno de los primeros en sancionar una ley de paridad a nivel nacional en América Latina y alcanzar resultados equilibrados en el acceso a la representación con la aplicación combinada de dos criterios ordenadores como son la paridad vertical y la paridad horizontal.

\section{La regla de la paridad y la violencia política contra las mujeres}

Archenti y Tula (2014) describían los alcances de los cambios de la legislación electoral en Bolivia y la inclusión de la regla de paridad para el armado de las listas electorales. Junto con Ecuador y Costa Rica, Bolivia constituye el grupo de los países pioneros en incorporar la paridad luego de la aplicación de las cuotas de género. 
En 2004 Bolivia incluyó la paridad para todos los cargos de representación popular con la aprobación de la Ley de Agrupaciones Ciudadanas y Pueblos Indígenas (2771/04). Establecía un piso «no menor al $50 \%$ para las mujeres» e introducía «la debida alternancia» para el ordenamiento de las candidaturas en las listas partidarias. Esta norma constituyó la base para posteriores legislaciones del país que fortalecieron el mecanismo de la paridad y alcanzar así los objetivos de igualdad en los órganos de representación.

Dos años después, la Ley Especial de Convocatoria a la Asamblea Nacional Constituyente reforzó la instrumentación de la paridad con el armado de las listas territoriales y departamentales bajo el sistema de «binomio y alternancia». El artículo 5 consideró que «la Asamblea Constituyente estaría conformada por 255 miembros, todos ellos iguales en derechos, jerarquías y obligaciones». De los tres candidatos a constituyente por circunscripción territorial, los dos primeros debían respetar la dupla «hombre-mujer» o «mujer-hombre» y en las circunscripciones departamentales con cinco candidatos, mínimamente dos debían ser mujeres, respetando la alternancia (artículos 15 y 16).

Respecto de la elección de los constituyentes, 210 debían ser elegidos en 70 circunscripciones trinominales con un sistema de reparto 2-1 (el partido ganador ocupa 2 asientos y el que sigue en votos 1) y 45 constituyentes electos en 9 circunscripciones pentanominales con la siguiente distribución 2-1-1-1. En caso de que la tercera y/o cuarta fuerza no obtuviera un porcentaje igual o mayor al $5 \%$ de los votos válidos, los constituyentes restantes se repartirían entre las dos primeras de acuerdo al residuo mayor obtenido. ${ }^{3}$

Los resultados de las elecciones del 2 de julio de 2006 mostraron el impacto positivo de la paridad con en el aumento de mujeres en la Cámara de Diputados y el 34\% de representación. ${ }^{4}$ De 255 constituyentes electos, 169 fueron hombres y 86 mujeres. El sistema paritario había duplicado los valores logrados con la aplicación de las cuotas de género del 30\%, cuyos porcentajes en la Cámara de Diputados habían oscilado del 19,2 en 2004 al 16,9 en $2006 .{ }^{5}$

\footnotetext{
${ }^{3}$ Artículo 14 de la ley especial de Convocatoria a la Asamblea Nacional Constituyente. ${ }^{4}$ Por las circunscripciones departamentales (plurinominales pentanominales) resultaron electos 34 hombres y 11 mujeres, mientras que en las circunscripciones territoriales (trinominales) los hombres ganaron 135 bancas y las mujeres, 75. En Gamboa Rocabado (2009). ${ }^{5}$ La ley 1779 o Ley de Reforma y Complementación al Régimen Electoral establecía un mínimo de 30\% de representación de mujeres en las listas, luego en 1999 se incorporó la
} 
La paridad no solo proporcionó un incremento cuantitativo sino también tuvo un importante impacto cualitativo. La constitución política del Estado Plurinacional de Bolivia promulgada por el presidente Evo Morales en febrero de 2009 destacó la participación de las mujeres y, además, una vieja deuda del estado boliviano para con los pueblos indígena originarios campesinos y su reconocimiento. Se crearon siete circunscripciones especiales uninominales para que los miembros de estos pueblos tuvieran representación en la Cámara de Diputados.

Respecto de la promoción de las mujeres en política, varios artículos que conforman esta nueva constitución hacen referencia a la «equivalencia de condiciones» e «igual participación entre hombres y mujeres» (artículos 8, 11, 26 y 147) fortaleciendo así las garantías de las normas anteriores sancionadas. Sin embargo, los términos «paridad» y «alternancia» solo aparecen mencionados explícitamente una sola vez cuando desarrolla la autonomía de los gobiernos departamentales y su forma de elección (artículo 278).

Por otro lado, la Ley 4021/09 o ley del Régimen Electoral Transitorio (LRET) reiteró en el artículo 9 la igualdad de oportunidades entre varones y mujeres para todos los cargos representativos (senadores, diputados titulares y suplentes, asambleístas departamentales, consejeros departamentales, concejales municipales y autoridades en los municipios). Y puso el acento en el sistema de alternancia entre candidatos/as titulares y suplentes tanto para cargos plurinominales como uninominales. Si el primer titular era hombre, debía seguir una titular mujer, una suplente mujer y luego un suplente varón (o viceversa). En el caso de las diputaciones uninominales la alternancia se expresaba entre titulares y suplentes de cada circunscripción. ${ }^{6}$

La nueva Asamblea Legislativa Plurinacional se inauguró asumiendo el mandato constitucional de aprobar, en un plazo máximo de 180 días, cinco leyes fundamentales. ${ }^{7}$ La Ley 026 del Régimen Electoral sancionada en 2010 fue una de ellas. Mucho más específica que las anterio-

\footnotetext{
alternancia. Sin embargo, la ausencia de sanción frente al incumplimiento perjudicó la efectividad de la norma, en Baldez y Brañez Cortés (2005).

${ }^{6}$ Esta ley regulaba el proceso electoral del 6 de diciembre de 2009 y del 4 de abril de 2010; además de los referendos autonómicos y la elección de asambleístas departamentales.

${ }^{7}$ Ley 18 del Órgano Electoral Plurinacional (16 de junio de 2010); Ley 25 del Órgano Judicial (24 de junio de 2010); Ley 26 del Régimen Electoral (30 de junio de 2010); Ley 27 del Tribunal Constitucional Plurinacional (6 de julio de 2010) y Ley Marco 31 de Autonomías y Descentralización «Andrés Ibáñez», (19 de julio de 2010).
} 
res, considera a la igualdad y equivalencia como principios de observancia obligatoria que rigen el ejercicio de la democracia intercultural (artículo 2). El artículo 11 refuerza por un lado la equivalencia de condiciones entre hombres y mujeres y, por el otro, obliga a las autoridades a garantizar y cumplir con la equidad de género y la igualdad de oportunidades según los criterios de alternancia y paridad.

A fin de que las candidaturas uninominales no sean mayoritariamente representadas por hombres, esta norma exige cumplir con al menos el $50 \%$ de titulares mujeres. Las listas provenientes de pueblos indígenas originarios campesinos deben respetar iguales criterios, mientras que para los cargos plurinominales se establece una preferencia hacia las mujeres cuando el número de escaños resulta impar.

Otra incorporación relevante es el reconocimiento del acoso político como delito electoral (artículo 238 inciso p) luego reconocido como delito penal en la Ley contra el acoso y violencia política hacia las mujeres (Ley 243/2012). Reitera que el estado garantiza el ejercicio pleno de los derechos políticos de las mujeres, generando las condiciones, oportunidades y medios que contribuyan a la participación equitativa y en igualdad de condiciones entre hombres y mujeres, aplicando la paridad y alternancia en la representación política en todas las entidades territoriales autónomas (artículo 6, d).

Cabe señalar que la ley recogió como actos de acoso y violencia política algunas de las maniobras adoptadas por los partidos políticos para evitar el cumplimiento de las cuotas/paridad, tales como: i) el hecho de proporcionar datos falsos o información incompleta de la identidad o sexo de quienes integran la lista; ii) divulgar o revelar información personal y privada de las candidatas electas, designadas con el objetivo de menoscabar su dignidad y obtener contra su voluntad la renuncia y/o licencia al cargo que ejercen o postulan.

\section{Composición de la Asamblea Legislativa y representación de las mujeres}

La Asamblea Legislativa Plurinacional es bicameral: los diputados son 130 (igual número que la anterior constitución), y los senadores 36 (antes eran 27). De acuerdo con la Ley de distribución de escaños entre Departamentos (2013) cambió la asignación de diputaciones volviendo a 60 los escaños plurinominales (que en 2010 eran 53) y a 63 los 
escaños uninominales (que en 2010 eran 70). Esta distribución se efectuó sobre la base del último Censo Nacional de Población y Vivienda del 21 de noviembre de 2012 para las elecciones generales de 2014 y se mantuvo para las elecciones generales de 2020.

El artículo 15 de la Ley 1266 del Régimen Excepcional y Transitorio para la Realización de Elecciones Generales 2020, aprobada por unanimidad en la Asamblea Legislativa Plurinacional, determinó que las elecciones generales de 2020 se efectuaran con la delimitación de circunscripciones publicada por el Órgano Electoral Plurinacional durante la gestión $2019 .{ }^{8}$

\section{Tabla 1: Cámara de Diputados. Cambios en la Magnitud de Distrito 2010-2014 \\ Bancas que se eligen por Departamento y Circunscripción}

\begin{tabular}{|c|c|c|c|c|c|c|c|}
\hline \multirow{4}{*}{ Departamentos } & \multicolumn{5}{|c|}{ Circunscripción } & \multicolumn{2}{|c|}{ Total } \\
\hline & \multicolumn{2}{|c|}{ Uninominal } & \multicolumn{2}{|c|}{ Plurinominal } & \multirow{2}{*}{ CEIOC } & \multicolumn{2}{|c|}{$\begin{array}{l}\text { diputados/as } \\
\text { departamento }\end{array}$} \\
\hline & 2010 & 2014 & 2010 & 2014 & & 2010 & 2014 \\
\hline & 6 & 5 & 5 & 5 & 0 & 11 & 10 \\
\hline La Paz & 15 & 14 & 13 & 14 & 1 & 29 & 29 \\
\hline Cochabamba & 10 & 9 & 8 & 9 & 1 & 19 & 19 \\
\hline Oruro & 5 & 4 & 3 & 4 & 1 & 9 & 9 \\
\hline Potosí & 8 & 7 & 6 & 6 & 0 & 14 & 13 \\
\hline Tarija & 5 & 4 & 3 & 4 & 1 & 9 & 9 \\
\hline Santa Cruz & 13 & 14 & 11 & 13 & 1 & 25 & 28 \\
\hline Beni & 5 & 4 & 3 & 3 & 1 & 9 & 8 \\
\hline Pando & 3 & 2 & 1 & 2 & 1 & 5 & 5 \\
\hline Total & 70 & 63 & 53 & 60 & 7 & & \\
\hline
\end{tabular}

Fuente: Elaboración propia sobre la base de Archenti y Tula (2014) y Atlas Electoral, 2017. Disponible en: https://www.oep.org.bo/wp-content/uploads/2017/04/ Atlas_Electoral_Tomo_IV.pdf

Sigla: CEIOC: Circunscripciones Especiales Indígenas Originario Campesinas

${ }^{8}$ Véase Boletín Informativo. Distribución y asignación de escaños. Órgano Electoral Plurinacional. Bolivia. 
La Tabla 1 muestra la redistribución de diputados/as por departamento, luego de la reforma electoral, sin alterar el número total de representantes (130). Desde 2014, sesenta y tres diputados se eligen por mayoría simple de votos en circunscripciones uninominales más las siete Circunscripciones Especiales Indígena Originario Campesinas (CEIOC) y, otra parte $(60)$, por representación proporcional ( $\mathrm{D}^{\prime} \mathrm{Hondt}$ ) distribuidos en nueve circunscripciones plurinominales variables, con un mínimo de 2 y un máximo de 16. Técnicamente 4 departamentos poseen magnitudes de distrito pequeña (inferior a 5), 3 son medianas (entre 6 y 10) y 2 grandes (más de 10) como Santa Cruz y La Paz (Nohlen, 1995).

El reparto de bancas en Diputados es de representación proporcional personalizado. Se efectúa tomando como base los votos plurinominales con un piso del $3 \%$ de los votos válidos a nivel nacional. Al total de bancas ganadas se adjudican primero las obtenidas en circunscripciones uninominales. Si quedaran bancas por cubrir, se incorporan proporcionalmente las de la lista plurinominal. Si el número de diputados elegidos en circunscripciones uninominales supera la proporción correspondiente se restan escaños plurinominales a los partidos que hayan obtenido los cocientes más bajos de votación (artículo 59, Ley 026/10)

Los senadores, se eligen de manera proporcional (fórmula $\mathrm{D}^{\prime}$ Hondt) en nueve circunscripciones tetranominales (4 por cada departamento). Tanto los diputados plurinominales como los senadores comparten la misma papeleta electoral junto con los cargos de presidente y vicepresidente.

Por su parte, las CEIOC se rigen por el principio de densidad poblacional en cada departamento. Solo están constituidas en las áreas rurales, y en aquellos departamentos donde estos grupos conformen una minoría poblacional (La Paz, Cochabamba, Oruro, Tarija, Santa Cruz, Beni y Pando). Los mandatos duran 5 años y pueden ser reelectos por una sola vez de manera continua.

\section{Las reglas de paridad en las elecciones de 2020}

En Bolivia, la paridad incorpora dos criterios ordenadores para armar las posiciones de las candidaturas en las listas electorales. La Norma Marco para Consolidar la Democracia Paritaria (2015) define a la Paridad Vertical (PV) o paridad de lista a la ubicación de las candidaturas de mujeres y hombres de manera alternativa y secuencial (uno a 
uno) en toda la extensión de la boleta electoral tanto en cargos titulares como suplentes. Mientras que Paridad Horizontal $(\mathrm{PH})$ o paridad territorial se entiende cuando una misma organización política y/o alianza se presenta en varios distritos electorales simultáneamente y deben acordar encabezamientos equitativos entre mujeres y hombres.

El Reglamento para la inscripción y registro de candidaturas emitido por el Tribunal Supremo Electoral (TSE-RSP-ADM 043/2020) reguló la inscripción de las candidaturas y el modo de presentación de listas para cumplir con la exigencia de la paridad vertical y paridad horizontal. En el capítulo 2 establece cómo debe ser la presentación de listas y contempla la posibilidad de listas completas, incompletas, pares e impares. La lista incompleta es cuando la organización política no cubre toda la nómina de candidaturas pudiendo quedar con número par o impar de miembros.

En el Senado se eligen cuatro senadores por cada uno de los nueve departamentos del país (en total 36). Así, si una organización política presenta una lista con candidaturas pares resulta indistinto quien ocupe el primer lugar. Pero, para garantizar la paridad a nivel nacional, al menos cinco departamentos deben llevar mujeres en la primera posición titular. Adicionalmente, si la cantidad de candidaturas titulares a senadoras/es presentadas por una organización política en un departamento es impar, las listas deben ser encabezadas por mujeres (artículo 9).

Los diputados también se eligen por departamento. Una parte en listas plurinominales variables y otra con listas uninominales. Para el caso de los diputados plurinominales, cuando se presenta un número par de candidaturas es indistinto quien encabeza la lista, pero cuando se trata de listas impares se debe dar preferencia a las mujeres (artículo 10). En los casos de circunscripciones uninominales, la igualdad, paridad y alternancia de género se expresa tanto en titulares como suplentes (artículo 11, ley 026). Sobre el total de circunscripciones delimitadas en cada departamento, las organizaciones políticas deben presentar, al menos, el cincuenta por ciento $(50 \%)$ de las candidaturas titulares con mujeres.

En las CIOC, que son siete, al menos cuatro deben tener a una mujer en la primera posición titular. Por último, entre otros requisitos formales el artículo 3, inciso g) señala que los candidatos no deben contar con antecedentes de violencia ejercida contra una mujer o contra cualquier miembro de su familia, con sentencia ejecutoriada en calidad de cosa juzgada. En el artículo 4, punto II, numeral 10 solicita a los candidatos que presenten un Certificado original y actualizado del Sistema 
Integral Plurinacional de Prevención, Atención, Sanción y Erradicación de la Violencia en razón de Género-SIPPASE, extendido por el Consejo de la Magistratura.

\section{Proceso electoral. Las elecciones generales del 18 de octubre de 2020}

Cuando se mide el impacto de las cuotas de género y/o de la paridad en el acceso a la representación de las mujeres deben considerarse, al menos, tres mecanismos institucionales que actúan coordinadamente Archenti y Tula $(2008,2010)$. Estas autoras señalaron: i) las características distintivas de cada diseño normativo, ii) el sistema electoral ${ }^{9}$ y iii) la cultura política. Por lo tanto, las evaluaciones que se efectúen sobre su real alcance e impactos sólo pueden ser tomadas observando el conjunto y nunca de manera aislada (Archenti y Tula 2008; Krook 2006; Norris y Lovenduski 1993).

Para las elecciones generales del 18 de octubre de 2020, el cronograma electoral establecía la fecha del 3 de febrero para registrar las candidaturas de las organizaciones políticas y alianzas. Según el Reporte Informativo 4 del Tribunal Supremo Electoral (TSE), fueron inscriptas en esta oportunidad 2.098 candidaturas al órgano legislativo, de las cuales 1.046 fueron mujeres y 1.052 varones. Según la legislación, las listas pueden ser modificadas posteriormente por inhabilitaciones o por renuncias hasta $72 \mathrm{hs}$. antes del día previsto para la elección general. Por lo tanto, la confirmación de las candidaturas se produce cuando el Órgano Electoral Plurinacional (OEP) publica en su portal el listado definitivo con las candidaturas habilitadas $24 \mathrm{hs}$. antes de la elección general. ${ }^{10}$

De las 8 formulas presidenciales presentadas inicialmente Frente Para la Victoria (FPV), Creemos, Libre 21, Comunidad Ciudadana (CC), Partido de Acción Nacional Boliviano (PANBOL), Juntos, Acción

\footnotetext{
${ }^{9}$ Una profusa bibliografía indica los efectos que tienen los diferentes componentes del sistema electoral para la efectividad de las cuotas de género, los cuales también aplican a la paridad. Al respecto léase, Norris (1985), Rule (1987), Caul (1999) y Htun \& Jones (2002).

${ }^{10}$ Calendario Electoral. Elecciones Generales 18 de octubre 2020. Aprobado por Resolución TSE-RSP-ADM 0193/2020 del 3 de agosto. Las actividades del calendario electoral se readecuaron luego de la suspensión temporal motivada por la pandemia. Disponible en: https://fuentedirecta.oep.org.bo/noticia/el-tse-aprueba-el-calendario-electoral-para-la-eleccion-del-18-de-octubre/
} 
Democrática Nacionalista (ADN) y Movimiento al Socialismo-Instrumento Político para la Soberanía del Pueblo (MAS-IPSP) hubo dos candidatas presidenciales (María de la Cruz Baya por ADN y Jeanine Añez por Juntos) y dos como vicepresidentas (Tomasa Yarhui por Libre 21 y Ruth Nina por PANBOL). Finalmente quedaron 5 listas para competir el 18 de octubre: MAS, CC, FPV, Creemos y PANBOL

En cuanto al armado de listas para la Asamblea Legislativa Plurinacional, el Órgano Electoral Plurinacional presentó varios reportes informativos para garantizar su efectivo cumplimiento. También realizó varios encuentros con autoridades partidarias a los efectos de difundir las reglamentaciones, evitar una incorrecta interpretación o su sanción.

Hacia el 13 de septiembre de 2020, el OEP publicó un documento de seguimiento en su sitio web (www.oep.org.bo) con el estado de cumplimiento de la paridad y alternancia de las organizaciones políticas. Pocos días antes de la elección, el TSE invitó a los delegados de las organizaciones políticas y alianzas a una reunión para garantizar que las listas finales cumplan con el requisito de paridad vertical y horizontal e identificar los eventuales ajustes que requieran efectuarse en cada organización.

En el Reporte Informativo 3/2020 del 12 de octubre titulado «Estado del cumplimiento de la paridad y alternancia en las listas de candidaturas» señaló que de 1.371 candidaturas habilitadas 684 eran de mujeres y 687 de varones. ${ }^{11}$ Expresó que -en términos generales- había cumplimiento pero cuando se revisaba cada organización política en particular, solo Comunidad Ciudadana había presentado cumplimiento total junto con las organizaciones de las naciones y pueblos indígena originario campesinos de diputaciones especiales. El seguimiento y monitoreo de organizaciones de la sociedad civil como la Coordinadora de la Mujer fue muy importante en todo este proceso.

El Órgano Electoral Plurinacional (OEP) publicó el 17 de octubre la Actividad 62 con el listado final de candidaturas habilitadas. ${ }^{12}$ Al día siguiente, el 18 de octubre, el OEP emitió un comunicado en el que se destacaba que todas las listas que competían en esa jornada electoral cumplían con los requisitos de paridad y alternancia ${ }^{13}$.

\footnotetext{
${ }^{11}$ Disponible en: https://www.oep.org.bo/wp-content/uploads/2020/10/Boletin _ListasParidad_EG_12_10_2020.pdf

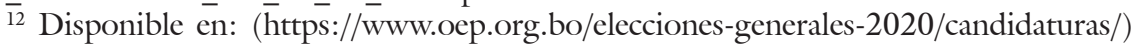

${ }^{13}$ Véase https://www.oep.org.bo/wpcontent/uploads/2020/10/Comunicado_TSE_18 _10_2020.jpg
} 


\section{Las mujeres y los números}

Las Tablas 2, 3, 4 y 5 muestran la proporción de género en ambas cámaras de la Asamblea Legislativa Plurinacional. En la Tabla 2 se observa el incremento de la participación política de las mujeres y su acceso a la representación tanto en los diputados/as plurinominales como en los uninominales. En estos últimos un aumento del $+47,5$ en 2014 como resultado de la primera aplicación de la legislación reforzada con la paridad horizontal.

Tabla 2: Participación de mujeres. Diputadas plurinominales y uninominales. En \%

\begin{tabular}{|l|c|c|c|c|c|c|}
\hline & $1997-2002$ & $2002-2005$ & $2005-2009$ & $2009-2014$ & 2014-2019 & 2020-2025 \\
\hline $\begin{array}{l}\text { Diputaciones } \\
\text { Plurinominales }\end{array}$ & 17,7 & 27,4 & 28,3 & 46,3 & 53,3 & 50 \\
\hline $\begin{array}{l}\text { Diputaciones } \\
\text { Uninominales }\end{array}$ & 2,9 & 10,3 & 7,1 & 1,7 & 49,2 & 49,2 \\
\hline
\end{tabular}

Fuente: Observatorio de Paridad Democrática del TSE, 2017. Para 2020, https:// computo.oep.org.bo/ y https://www.oep.org.bo/elecciones-generales-2020/candidaturas/ Nota: En diputaciones uninominales se cuentan también las ganadas por mujeres en las CEIOC.

En las elecciones 2020 Bolivia alcanzó el 46,9\% de mujeres en la Cámara de Diputados. Un total de 61 diputadas cuando se suman las mujeres ingresantes por las diferentes arenas de representación: plurinominales, 30; uninominales, 27 y por las CEIOC, 4 (Ver Tablas 5, 6 y 7). Este resultado es inferior al alcanzado en 2014 cuando por primera vez se logró el 50\% como consecuencia del aumento en los encabezamientos de listas de mujeres en los distritos uninominales.

Tabla 3: Total Mujeres Cámara de Diputados

\begin{tabular}{|l|l|l|l|l|l|l|l|}
\hline & \multicolumn{7}{|c|}{ Años } \\
\cline { 2 - 8 } & 1993 & 1997 & 2000 & 2001 & 2002 & 2003 & 2004 \\
\hline 10 & 11.5 & 11.5 & 11.5 & 18.5 & 18.5 & 19.2 \\
\hline
\end{tabular}

Fuente: CEPAL y Observatorio de Género. Coordinadora de la Mujer. Bolivia. 


\section{Tabla 4: Total Mujeres en Cámara de Diputados 2005-2020 (en \%)}

\begin{tabular}{|r|l|l|l|l|l|l|l|l|}
\hline \multirow{2}{*}{} & \multicolumn{9}{|c|}{ Años } \\
\cline { 2 - 9 } & 2005 & 2006 & 2007 & 2008 & 2009 & 2010 & 2014 & 2020 \\
\hline$\%$ & 16.9 & 16.9 & 16.9 & 16.9 & 22.3 & 25.4 & 50 & 46,9 \\
\hline
\end{tabular}

Fuente: CEPAL y Observatorio de Género. Coordinadora de la Mujer. Bolivia.

Bolivia ocupa así el décimo lugar entre los países que poseen cuotas de género y/o paridad para la elección de sus representantes en la cámara de diputados o cámaras únicas del mundo. En América Latina está por debajo de Cuba $(53,4 \%)$, Nicaragua $(48,4 \%)$ y México $(48,2 \%)^{14}$, los dos últimos incluyen paridad para la elección de sus cargos legislativos.

Tabla 5: Proporción de puestos ocupados por mujeres Cámara de Senadores 1982-2020 (en \%)

\begin{tabular}{|l|l|l|l|l|l|l|l|l|l|l|}
\hline & \multicolumn{10}{|c|}{ Años } \\
\cline { 2 - 11 } & 1982 & 1985 & 1989 & 1993 & 1997 & $\mathbf{2 0 0 2}$ & $\mathbf{2 0 0 5}$ & $\mathbf{2 0 0 9}$ & $\mathbf{2 0 1 4}$ & $\mathbf{2 0 2 0}$ \\
\hline & 7.4 & 0 & 3.7 & 3.7 & 3.7 & 14.8 & 3.7 & 44.4 & 44.4 & 55.6 \\
\hline
\end{tabular}

Fuente: Observatorio de Género. Coordinadora de la Mujer. Bolivia. En: http:// www.coordinadoradelamujer.org.bo Para 2020, https://computo.oep.org.bo/ y https:// www.oep.org.bo/elecciones-generales-2020/candidaturas/

Nota: Hasta 2005, el total de senadores era de 27, en 2009 con la reforma constitucional pasó a 36 miembros.

En la Cámara de Senadores las elecciones de 2020 llegaron al record histórico del $55,6 \%$ y un senado conformado por primera vez con más mujeres que varones.

\section{Con la lupa en los departamentos y circunscripciones}

Las tablas que siguen muestran el cruce de información de dos sitios oficiales que permiten arribar al dato de las mujeres ingresantes

${ }^{14}$ En Interparliamentary Union. Véase: https://data.ipu.org/women-ranking?month= $1 \& y e a r=2021$ 
en la Asamblea Legislativa Plurinacional para el periodo 2020-2025 y los votos recibidos en las listas uninominales y la que lleva a presidente, senadores y diputados plurinominales conjuntamente. Así, tomando los resultados electorales del sitio oficial https://computo.oep.org.bo/ y las candidaturas oficializadas publicadas el 17 de septiembre en https:// www.oep.org.bo/elecciones-generales-2020/candidaturas/ se ha logrado identificar los lugares donde las candidatas ganaron asientos parlamentarios. Se observó: i) cada categorías en juego (diputados uninominales, diputados plurinominales y diputados especiales), ii) los votos recibidos por cada organización política, iii) los cargos ganados en función de los votos recibidos y iv) la cantidad de mujeres electas.

\section{Tabla 6: Diputaciones uninominales. Mujeres Electas por circunscripción}

\begin{tabular}{|c|c|c|c|c|}
\hline \multicolumn{5}{|c|}{ Diputados Uninominales } \\
\hline \multirow[b]{2}{*}{$\begin{array}{l}\text { Departamento } \\
\text { y número de } \\
\text { bancas }\end{array}$} & \multirow[b]{2}{*}{ Circunscripción } & \multicolumn{2}{|c|}{ Resultados } & \multirow{2}{*}{\begin{tabular}{|c|} 
Total de \\
Mujeres \\
Electas por \\
Departamentc
\end{tabular}} \\
\hline & & $\begin{array}{c}\text { Partido Político } \\
\text { Ganador } \%\end{array}$ & $\begin{array}{c}\text { Mujeres } \\
\text { Titulares Electas } \\
\text { por Partido } \\
\text { Político }\end{array}$ & \\
\hline \multirow{5}{*}{$\begin{array}{c}\text { Chuquisaca } \\
\text { (5) }\end{array}$} & 1 & CC: 58,55 & -- & \multirow{5}{*}{2} \\
\hline & 2 & CC: 50,85 & SI & \\
\hline & 3 & $\begin{array}{l}\text { MAS-IPSP: } \\
85,99\end{array}$ & -- & \\
\hline & 4 & $\begin{array}{l}\text { MAS-IPSP: } \\
68,59\end{array}$ & SI & \\
\hline & 5 & $\begin{array}{l}\text { MAS-IPSP: } \\
80,97\end{array}$ & -- & \\
\hline \multirow{8}{*}{$\begin{array}{l}\text { La Paz } \\
(14)\end{array}$} & 6 & CC: 51,36 & -- & \multirow{8}{*}{6} \\
\hline & 7 & CC: 53,99 & -- & \\
\hline & 8 & CC: 51,22 & -- & \\
\hline & 9 & $\begin{array}{l}\text { MAS-IPSP: } \\
49,64\end{array}$ & -- & \\
\hline & 10 & $\begin{array}{l}\text { MAS-IPSP: } \\
75,68\end{array}$ & SI & \\
\hline & 11 & $\begin{array}{l}\text { MAS-IPSP: } \\
61,60\end{array}$ & -- & \\
\hline & 12 & $\begin{array}{l}\text { MAS-IPSP: } \\
83,66\end{array}$ & SI & \\
\hline & 13 & $\begin{array}{l}\text { MAS-IPSP: } \\
81,49\end{array}$ & -- & \\
\hline
\end{tabular}




\begin{tabular}{|c|c|c|c|c|}
\hline \multirow{6}{*}{$\begin{array}{c}\text { La Paz } \\
(14)\end{array}$} & 14 & $\begin{array}{l}\text { MAS-IPSP: } \\
91,21\end{array}$ & -- & \\
\hline & 15 & $\begin{array}{l}\text { MAS-IPSP: } \\
84,97\end{array}$ & SI & \\
\hline & 16 & $\begin{array}{l}\text { MAS-IPSP: } \\
78,68\end{array}$ & -- & \\
\hline & 17 & $\begin{array}{l}\text { MAS-IPSP: } \\
71,54\end{array}$ & SI & \\
\hline & 18 & $\begin{array}{l}\text { MAS-IPSP: } \\
90,44\end{array}$ & SI & \\
\hline & 19 & $\begin{array}{l}\text { MAS-IPSP: } \\
83,63\end{array}$ & SI & \\
\hline \multirow{9}{*}{$\begin{array}{c}\text { Cochabamba } \\
\text { (9) }\end{array}$} & 20 & CC: 68,63 & -- & \multirow{9}{*}{4} \\
\hline & 21 & $\begin{array}{l}\text { MAS-IPSP: } \\
62,39\end{array}$ & -- & \\
\hline & 22 & $\begin{array}{l}\text { MAS-IPSP: } \\
59,90\end{array}$ & SI & \\
\hline & 23 & $\begin{array}{l}\text { MAS-IPSP: } \\
64,85\end{array}$ & SI & \\
\hline & 24 & $\begin{array}{l}\text { MAS-IPSP: } \\
97,76\end{array}$ & -- & \\
\hline & 25 & $\begin{array}{l}\text { MAS-IPSP: } \\
92,03\end{array}$ & -- & \\
\hline & 26 & $\begin{array}{l}\text { MAS-IPSP: } \\
80,82\end{array}$ & SI & \\
\hline & 27 & $\begin{array}{l}\text { MAS-IPSP: } \\
77,51\end{array}$ & SI & \\
\hline & 28 & $\begin{array}{l}\text { MAS-IPSP: } \\
55,47\end{array}$ & -- & \\
\hline \multirow{4}{*}{$\begin{array}{l}\text { Oruro } \\
(4)\end{array}$} & 29 & $\begin{array}{l}\text { MAS-IPSP: } \\
52,21\end{array}$ & SI & \multirow[t]{4}{*}{2} \\
\hline & 30 & $\begin{array}{l}\text { MAS-IPSP: } \\
48,29\end{array}$ & -- & \\
\hline & 31 & $\begin{array}{l}\text { MAS-IPSP: } \\
81,88\end{array}$ & SI & \\
\hline & 32 & $\begin{array}{l}\text { MAS-IPSP: } \\
85,98\end{array}$ & -- & \\
\hline \multirow{7}{*}{$\begin{array}{l}\text { Potosí } \\
\text { (7) }\end{array}$} & 33 & CC: 63,98 & -- & \multirow{7}{*}{3} \\
\hline & 34 & CC: 52,87 & SI & \\
\hline & 35 & $\begin{array}{l}\text { MAS-IPSP: } \\
80,01\end{array}$ & -- & \\
\hline & 36 & $\begin{array}{l}\text { MAS-IPSP: } \\
67,03\end{array}$ & SI & \\
\hline & 37 & $\begin{array}{l}\text { MAS-IPSP: } \\
70,23\end{array}$ & -- & \\
\hline & 38 & $\begin{array}{l}\text { MAS-IPSP: } \\
80,72\end{array}$ & SI & \\
\hline & 39 & $\begin{array}{l}\text { MAS-IPSP: } \\
71,34\end{array}$ & -- & \\
\hline
\end{tabular}




\begin{tabular}{|c|c|c|c|c|}
\hline \multirow{4}{*}{$\begin{array}{c}\text { Tarija } \\
(4)\end{array}$} & 40 & CC: 45,49 & $\begin{array}{l}- \\
\end{array}$ & \multirow{4}{*}{2} \\
\hline & 41 & CC: 53,86 & SI & \\
\hline & 42 & $\begin{array}{l}\text { MAS-IPSP: } \\
47,25\end{array}$ & -- & \\
\hline & 43 & $\begin{array}{l}\text { MAS-IPSP: } \\
40,18\end{array}$ & SI & \\
\hline \multirow{14}{*}{$\begin{array}{c}\text { Santa Cruz } \\
\text { (14) }\end{array}$} & 44 & $\begin{array}{l}\text { CREEMOS: } \\
70,60\end{array}$ & -- & \multirow{14}{*}{5} \\
\hline & 45 & $\begin{array}{l}\text { CREEMOS: } \\
77,38\end{array}$ & -- & \\
\hline & 46 & $\begin{array}{l}\text { CREEMOS: } \\
56,65\end{array}$ & SI & \\
\hline & 47 & $\begin{array}{l}\text { CREEMOS: } \\
56,52\end{array}$ & SI & \\
\hline & 48 & $\begin{array}{l}\text { CREEMOS: } \\
46,43\end{array}$ & -- & \\
\hline & 49 & $\begin{array}{l}\text { MAS-IPSP: } \\
50,69\end{array}$ & -- & \\
\hline & 50 & $\begin{array}{l}\text { CREEMOS: } \\
49,36\end{array}$ & -- & \\
\hline & 51 & $\begin{array}{l}\text { CREEMOS: } \\
62,80\end{array}$ & -- & \\
\hline & 52 & $\begin{array}{l}\text { MAS-IPSP: } \\
47,24\end{array}$ & SI & \\
\hline & 53 & $\begin{array}{l}\text { MAS-IPSP: } \\
57,68\end{array}$ & SI & \\
\hline & 54 & $\begin{array}{l}\text { CREEMOS: } \\
57,67\end{array}$ & SI & \\
\hline & 55 & $\begin{array}{l}\text { MAS-IPSP: } \\
44,06\end{array}$ & -- & \\
\hline & 56 & $\begin{array}{l}\text { MAS-IPSP: } \\
51,52\end{array}$ & -- & \\
\hline & 57 & $\begin{array}{l}\text { MAS-IPSP: } \\
50,51\end{array}$ & -- & \\
\hline \multirow{4}{*}{$\begin{array}{l}\text { Beni } \\
(4)\end{array}$} & 58 & $\begin{array}{l}\text { CREEMOS: } \\
34,93\end{array}$ & -- & \multirow[t]{4}{*}{2} \\
\hline & 59 & MAS-IPSP: 51,90 & SI & \\
\hline & 60 & CC: 37,11 & SI & \\
\hline & 61 & $\begin{array}{l}\text { CREEMOS: } \\
35,77\end{array}$ & -- & \\
\hline \multirow{2}{*}{$\begin{array}{c}\text { Pando } \\
(2)\end{array}$} & 62 & $\begin{array}{l}\text { MAS-IPSP: } \\
42,07\end{array}$ & SI & \multirow[t]{2}{*}{1} \\
\hline & 63 & $\begin{array}{l}\text { MAS-IPSP: } \\
55,28\end{array}$ & -- & \\
\hline Total & \multicolumn{4}{|c|}{$\frac{1}{27 / 63}$} \\
\hline
\end{tabular}

Fuente: Elaboración propia sobre datos oficiales de https:/computo.oep.org.bo/ y https://www.oep.org.bo/elecciones-generales-2020/candidaturas/.

Siglas: CC. Comunidad Ciudadana, MAS-IPSP: Movimiento al SocialismoInstrumento Político para la Soberanía del Pueblo. 


\section{Tabla 7: Diputaciones plurinominales y Senado. Mujeres Electas por Departamento}

\begin{tabular}{|c|c|c|c|c|c|c|}
\hline Departamentos & $\begin{array}{c}\text { Partidos } \\
\text { Políticos/Alianzas } \\
\text { Electorales }\end{array}$ & \begin{tabular}{|c|} 
Votos $\%$ \\
Presidente
\end{tabular} & $\begin{array}{c}\text { Diputados } \\
\text { Plurinominales }\end{array}$ & Mujeres & Senadores & Mujeres \\
\hline \multirow{3}{*}{ Chuquisaca } & \multirow{3}{*}{ MAS-IPSP } & \multirow[b]{2}{*}{49,06} & MD: 5 & \multirow[b]{2}{*}{1} & MD: 4 & \multirow{3}{*}{1} \\
\hline & & & 2 & & 2 & \\
\hline & & 45,99 & 3 & 2 & 2 & \\
\hline \multirow[b]{3}{*}{$\mathrm{La} \mathrm{Paz}$} & \multirow{3}{*}{ MAS-IPSP } & \multirow[b]{2}{*}{68,36} & MD: 14 & \multirow[b]{2}{*}{4} & MD: 4 & \multirow[b]{2}{*}{2} \\
\hline & & & 9 & & 3 & \\
\hline & & 28,58 & 5 & 2 & 1 & 1 \\
\hline \multirow[b]{3}{*}{ Cochabamba } & \multirow[b]{2}{*}{ MAS-IPSP } & \multirow[b]{2}{*}{65,90} & MD: 9 & \multirow[b]{2}{*}{2} & MD: 4 & \multirow[b]{2}{*}{1} \\
\hline & & & 4 & & 3 & \\
\hline & $\mathrm{CC}$ & 31,68 & 5 & 3 & 1 & 1 \\
\hline \multirow[b]{3}{*}{ Oruro } & \multirow{3}{*}{ MAS-IPSP } & \multirow{3}{*}{$\begin{array}{r}62,94 \\
-33,02\end{array}$} & MD: 4 & \multirow{3}{*}{1} & MD: 4 & \multirow{3}{*}{1} \\
\hline & & & 1 & & 3 & \\
\hline & & & 3 & & 1 & \\
\hline \multirow[b]{3}{*}{ Potosí } & \multirow{3}{*}{ MAS-IPSP } & \multirow[b]{2}{*}{57,61} & MD: 6 & \multirow[b]{2}{*}{2} & MD: 4 & \multirow[b]{2}{*}{1} \\
\hline & & & 3 & & 3 & \\
\hline & & 35,85 & 3 & 1 & 1 & 1 \\
\hline \multirow[b]{3}{*}{ Tarija } & \multirow{3}{*}{\begin{tabular}{c} 
CC \\
\hdashline$M A Y-I P S P$
\end{tabular}} & \multirow{3}{*}{$\begin{array}{l}50,24 \\
41,62\end{array}$} & MD: 4 & \multirow[b]{2}{*}{1.} & MD: 4 & \multirow{3}{*}{1} \\
\hline & & & 2. & & 2 & \\
\hline & & & 2 & 1 & 2 & \\
\hline & & & MD: 13 & & MD: 4 & \\
\hline Santa Cruz & CREEMOS & 45,08 & 5 & 3 & 2 & 1. \\
\hline & MÄS-IPSP & 36,21 & $\because 4$ & 2 & 2 & 1 \\
\hline & $\mathrm{CC}$ & 17,32 & 4 & 2 & & -- \\
\hline & & & MD: 3 & & MD: 4 & \\
\hline & CC & 39,17 & 2 & 1 & 2 & 1 \\
\hline Beni & MAS-IPSP & 34,72 & 1 & 1 & 1 & 1 \\
\hline & CREEMOOS & 23,70 & $\cdots$ & (-- & 1 & 1 \\
\hline & & & MD: 2 & & MD: 4 & \\
\hline & MAS-IPSP & 45,80 & $\begin{array}{c}0- \\
-\cdots\end{array}$ & --- & 2 & 1 \\
\hline Pando & CREEMOS & 26,22 & 1 & -- & 1 & -- \\
\hline & $\overline{C C}$ & 25,89 & 1 & -- & 1 & 1 \\
\hline & & $\begin{array}{l}\text { Total } \\
\end{array}$ & 60 & 30 & 36 & 20 \\
\hline
\end{tabular}

Fuente: Elaboración propia sobre datos oficiales de https://computo.oep.org.bo/ y https://www.oep.org.bo/elecciones-generales-2020/candidaturas/

Siglas: CC. Comunidad Ciudadana, MAS-IPSP: Movimiento al SocialismoInstrumento Político para la Soberanía del Pueblo. 
Tabla 8: Diputaciones Especiales. Mujeres Electas por departamento

\begin{tabular}{|l|c|c|c|}
\hline \multirow{4}{*}{\begin{tabular}{|}
\multirow{4}{*}{$\begin{array}{l}\text { Circunscripción } \\
\text { Especiales }\end{array}$} \\
\cline { 2 - 4 }
\end{tabular}} & $\begin{array}{c}\text { Partido Político } \\
\text { y \% }\end{array}$ & Mujeres Electas \\
\cline { 2 - 4 } & La Paz & MAS-IPSP 55,85 & Verónica Challco \\
\cline { 2 - 4 } & Cochabamba & MAS-IPSP 98,44 & -- \\
\cline { 2 - 4 } & Oruro & MAS-IPSP 75,12 & -- \\
\cline { 2 - 4 } & Tarija & MAS-IPSP 30,58 & Darlen Velasco Torrez \\
\cline { 2 - 4 } & Santa Cruz & MAS-IPSP 44,44 & Elsa Sanchez Romero \\
\cline { 2 - 4 } & Beni & MAS-IPSP 77,17 & Tacni Mendoza \\
\cline { 2 - 4 } & Pando & MAS-IPSP 73,07 & Tados \\
\hline
\end{tabular}

Fuente: Elaboración propia sobre datos oficiales de https://computo.oep.org.bo/ y https:/www.oep.org.bo/elecciones-generales-2020/candidaturas/

Como se desprende de las Tablas 5, 6 y 7 de los 63 diputados uninominales, 27 son mujeres (20 del MAS-IPSP, 4 de CC y 3 de CREEMOS). Si sumamos las 4 bancas de las diputaciones especiales se llega al $49,2 \%$ de mujeres electas por circunscripción. Lo importante de observar es que todos los departamentos poseen representantes mujeres y esto es un efecto directo de la legislación que combina la paridad vertical con la paridad horizontal.

En cuanto al cargo de diputado plurinacional, éstos se incorporan una vez que se han cubierto los cargos uninominales ganados y quedan aún escaños por cubrir del total general obtenido. Por esta razón en la Tabla 6 no hay una relación directa entre los votos obtenidos por una agrupación política y los cargos ganados, sino que ésta última depende de cuántos asientos haya ganado ya esa agrupación política en la circunscripción uninominal. De las 60 bancas, las mujeres ganaron 30. El MASIPSP aportó 15 diputadas, CC 12 y CREEMOS 3. Con 30 mujeres, el porcentaje alcanzado es del $50 \%$. En esta categoría también se observa un retroceso de $-3,3 \%$ respecto de 2014 cuando se había obtenido el $53,3 \%$.

En el caso de los Diputados Especiales, todas las circunscripciones han sido ganadas por el MAS-IPSP aun cuando -en esta oportunidadlas comunidades indígena originario campesinas podían postular candidaturas propias sin la necesidad de contar con un aval partidario. 
Para el Senado el reparto ha sido de 20 bancas para las mujeres sobre 36. El MAS-IPSP fue la organización política que más mujeres aportó con 10 bancas, mientras que CC obtuvo 8 y CREEMOS 2 . El porcentaje alcanzado es del 55,6\%. Este resultado también se explica por la obligación legal de postular a mujeres en primer lugar titular en, al menos, la mitad de los departamentos. A diferencia de lo que ocurre en la Cámara de Diputados, el Senado tiene un incremento de mujeres de $+11,2 \%$ respecto de 2014 cuando sacó el 44,4\%.

\section{A modo de conclusión}

La paridad de género en el ámbito político-electoral busca equiparar la participación de las mujeres en los órganos de representación. Las organizaciones políticas son centrales no solo porque expresan un canal de comunicación entre el estado y la sociedad sino también porque tradicionalmente han asumido ( $\mathrm{y}$ en algunos países con exclusividad) el monopolio de las candidaturas en el acceso a la representación.

Como se observa en este trabajo, las legislaciones dictadas en Bolivia durante el período en estudio han ido fortaleciendo los diversos mecanismos institucionales para lograr que la paridad no sólo se cumpla en la oferta electoral sino que tenga impacto positivo en el acceso a la representación. Las estrategias combinadas de paridad vertical y paridad horizontal permiten el cumplimiento de los objetivos de una mayor equidad en los órganos de representación con la alternancia en los encabezamientos de lista y/o dando preferencia a las mujeres en las primeras posiciones titulares.

\section{Referencias bibliográficas}

Archenti, N. y Tula, M. I. (Eds.). (2008). Mujeres y Politica en América Latina. Sistemas Electorales y Cuotas de Género. Buenos Aires, Argentina: Heliasta.

Archenti, N. y Tula, M. I. (2010). (Des) Igualdad de género en las listas partidarias. Argentina 2007. En Ansaldi, W., Funes, P., y Villavicencio, S., (Comps.), Bicentenario, otros relatos, (pp. 253-272). Buenos Aires, Argentina: Instituto de Investigaciones Gino GermaniInstituto de América Latina y el Caribe. 
Archenti, N. y Tula, M. I. (2014). De las cuotas a la paridad en América Latina: los casos de Bolivia, Ecuador y Costa Rica. En Archenti, N. y Tula, M. I. (Coords). La representación imperfecta. Logros y desafios de las mujeres politicas, (pp.13-36). Buenos Aires, Argentina: EUDEBA.

Archenti, N. y Tula, M. I. (2017). Critical Challenges of Quotas and Parity in Latin America. En Dosek, T. et. al. Women, Politics and democracy in Latin American, (pp. 29-44). Londres, Reino Unido: Palgrave.

Baldez, L. y Brañez Cortés, P. (2005). ¿Cuánto hemos avanzado las mujeres con las cuotas? El caso boliviano. En León, M. (Ed) Nadando contra la corriente. Mujeres y cuotas en los paises andinos. Quito, Ecuador: Flacso-Ecuador.

Choque Aldana, M. (2013). Paridad y alternancia en Bolivia. Avances y desafíos de la participación de las mujeres en la política. En CIMOEA-IDEA INTERNACIONAL. La apuesta por la paridad: democratizando el sistema politico en América Latina. Los casos de Ecuador, Bolivia y Costa Rica.

Gamboa Rocabado, F. (2009). Dilemas y Conflictos sobre la Constitución de Bolivia. Historia Política de la Asamblea Constituyente. La Paz, Bolivia: Konrad Adenauer Stiftung.

Htun, M. y Jones, M. (2002). Engendering the right to participate in decisión-making electoral quotas in women's leadership in Latin America. En Craske, N. y Molineux, M. Gender and the politics of rights and democracy in Latin America, (pp.32-56). Londres, Reino Unido: Palgrave.

Johnson, N. (2014). Las barreras que la cuota no superó: selección de candidaturas y desigualdades de género en América Latina. En Archenti, N. y Tula, M. I. Tula (Coords.). La representación imperfecta. Logros y desafios de las mujeres politicas. Buenos Aires, Argentina: EUDEBA.

Krook, M. L. (2006). Quotas for women in politics. Gender and candidate selection reform worldwide. Oxford, Reino Unido: Oxford University Press.

ONU Mujeres (2016). Norma Marco para Consolidar una democracia paritaria.

Nohlen, D. (1994). Sistemas electorales y partidos politicos. México, Méxi- 
co: Fondo de Cultura Económico.

Norris, P. (1985). Women's Legislative Participation in Western Europe. West European Politics 8 (4), 90-101.

Norris, P. y Lovedunski, J. (Eds.). (1993). Gender and Party Politics. Londres, Reino Unido: Sage.

Rule, W. (1987). Electoral Systems, Contextual Factors and Women's Opportunity for Election to Parliament in Twenty-Three Democracies. Western Political Quarterly, 40 (3), 477-498.

Saba, R. (2016). Más allá de la igualdad formal ante la ley. ¿Qué les debe el estado a los grupos desaventajados? Buenos Aires, Argentina: Siglo XXI.

Tula, M. I. (2017) .Paridad de género y acceso a la representación política. ¿Por qué? ¿Para qué? En Blando, O. (Comp.). Reforma política en Argentina: un debate democrático pendiente, (pp. 109-124). Rosario, Argentina: UNR Editora. 